\section{Análise da qualidade da informação autorreferida sobre duração do sono de escolares do Estudo de Riscos Cardiovasculares em Adolescentes (ERICA)}

\author{
Quality analysis of self-reported information on \\ sleep duration in schoolchildren in the Study of \\ Cardiovascular Risk Factors in Adolescents (ERICA)
}

\author{
Análisis sobre calidad de los datos autoinformados \\ sobre la duración del sueño en escolares del \\ Estudio de Riesgos Cardiovasculares en \\ Adolescentes (ERICA)
}

QUESTÕES METODOLÓGICAS METHODOLOGICAL ISSUES
Gabriela de Azevedo Abreu 1

Thiago Luiz Nogueira da Silva 2

Liliane Reis Teixeira 3

Katia Vergetti Bloch 2

\title{
Resumo
}

Objetivou-se avaliar o comportamento do sono de adolescentes de 12 a 17 anos, participantes do ERICA (Estudo de Riscos Cardiovasculares em Adolescentes), estudo de representatividade nacional, segundo características sociodemográficas, turno e tipo de escola, e descrever a correção das horas inconsistentes. A coleta de dados ocorreu em 2013 e 2014. Foram feitas quatro perguntas sobre horas habituais de dormir e de acordar durante a semana e no final de semana, com 24 opções de respostas, uma para cada hora do dia. A análise das inconsistências considerou a distribuição de frequências das respostas quanto às horas habituais de dormir e de acordar, além da compatibilidade com o turno. A duração do sono durante a semana e final de semana foi obtida pela diferença entre horas habituais de dormir e de acordar e foi excluída se $\leq 4$ ou $\geq 14$ horas. A média de sono total da semana foi calculada pela fórmula: (duração do sono durante a semana $x 5+$ duração do sono no final de semana $x$ 2)/7. Foram criados três grupos: dados originais (respostas que não necessitaram correções), dados corrigidos (inconsistentes, mas passíveis de correção) e dados excluídos (inconsistentes, para os quais não se tinha critério para correção). A correção recuperou informação inconsistente de 5.988 adolescentes (8\%) dos 74.589 participantes. Foram excluídas $7.937(10,6 \%)$ respostas. Os adolescentes cujas informações foram corrigidas ou excluídas são mais novos, do sexo masculino, de escolas públicas e da Região Norte. A correção minimizou perdas e conferiu maior consistência ao tratamento dos dados. O estudo contribui para o aprimoramento da construção de instrumentos de coleta de dados em estudos observacionais, tornando transparente a forma de lidar com as limitações inerentes ao método de coleta de dados.

Correspondência

T. L. N. Silva

Instituto de Estudos em Saúde Coletiva, Universidade Federal do Rio de Janeiro.

Av. Horácio Macedo s/n, Próximo a Prefeitura Universitária da UFRJ, Rio de Janeiro, RJ 21941-598, Brasil.

thiagolns@gmail.com

1 Instituto de Medicina Social, Universidade do Estado do Rio de Janeiro, Rio de Janeiro, Brasil.

2 Instituto de Estudos em Saúde Coletiva, Universidade Federal do Rio de Janeiro, Rio de Janeiro, Brasil.

3 Escola Nacional de Saúde Pública Sergio Arouca, Fundação Oswaldo Cruz, Rio de Janeiro, Brasil. 


\section{Introdução}

A adolescência é uma fase da vida marcada por mudanças biopsicossociais importantes, inclusive em relação ao padrão do ciclo vigília/sono. Atualmente, os adolescentes têm cada vez mais apresentado horários tardios de dormir devido ao uso de aparelhos eletrônicos como computadores e telefones, participação em eventos sociais, o que os leva a dormir tarde e, como precisam acordar cedo para as aulas da manhã, acabam tendo uma importante diminuição das horas de sono ${ }^{1}$. Por conseguinte, uma duração inadequada de sono está associada a um aumento da sonolência diurna, dificuldades de aprendizagem e diminuição da qualidade de vida 2,3,4. Além disso, alguns estudos apontam que os adolescentes que dormem pouco tendem a apresentar alterações no perfil lipídico 5,6 e aumento de peso 2,6,7. Assim, a duração do sono tornou-se um fator de risco potencialmente importante e inovador para a investigação também de doenças crônicas em estudos epidemiológicos 8 .

A avaliação do sono, de forma global, envolve a análise de quantidade, qualidade, arquitetura e de comportamentos do sono 9,10, e pode ser realizada com a utilização de vários instrumentos, com medidas envolvendo aspectos subjetivos e objetivos 11. O estudo polissonográfico (PSG) ainda é considerado o instrumento de referência para o diagnóstico da maioria dos transtornos do sono e também para o conhecimento da efetiva duração do sono, em horas 10,12,13. Mais recentemente, vem sendo muito utilizado em pesquisas e na prática clínica sensores de movimento capazes de determinar períodos de atividade e repouso, com base em uma técnica conhecida como actimetria (ACT) em aparelhos similares a relógios de pulso, validada utilizando-se o PSG como padrão-ouro ${ }^{14}$. Os sensores de movimento são considerados como uma alternativa mais simples, não invasiva, prática e de menor custo ao PSG e podem ser usados em estudos populacionais 15.

Devido ao alto custo e pela dificuldade da utilização do PSG e de outros métodos objetivos de avaliação do sono, questionários são usados em estudos epidemiológicos como métodos alternativos no intuito de se obter informações sobre o sono do indivíduo de forma rápida, simples, padronizada, com menores custos 16 e de forma repetida em um número elevado de indivíduos 17. Entretanto, as informações coletadas por meio de respostas aos questionários são chamadas de medidas indiretas porque são dependentes de condições inerentes aos sujeitos, tais como memória, capacidade de compreensão do que está sendo perguntado, além de serem mediadas por interesses dos próprios entrevistados, como a própria motivação em querer responder e de fazê-lo de forma acurada. Dessa forma, é muito importante que as questões sejam elaboradas de maneiras mais simples e objetivas possíveis, a fim de evitar problemas de compreensão, o que depende muito dos padrões culturais dos sujeitos entrevistados 18 .

Devido à existência de possíveis desvantagens no uso de questionários em estudos epidemiológicos, alguns autores apontam a necessidade de detalhamento sobre as possíveis fontes de erros sistemáticos na tentativa de evitar ou minimizar vieses nesses estudos 19,20,21.

Questões relacionadas a vieses de informação têm sido pouco enfatizadas na prática e, até de certa forma, encaradas com descaso por muitos pesquisadores, principalmente em relação à avaliação da duração do sono via aplicação de questionários. Não se menciona nos estudos as inconsistências dos dados coletados de duração do sono, em especial os de autopreenchimento, e qual estratégia foi ou deve ser adotada para corrigi-las quando existem. Frequentemente, ênfase é dada aos problemas relacionados aos desenhos de estudo e à análise de dados, mas desenhos ou análises estatísticas sofisticadas não produzem resultados mais confiáveis quando a qualidade das informações coletadas é insatisfatória 22,23. Uma vez ocorrida a falha na obtenção da informação, seja devido a problemas no instrumento, na coleta dos dados em si, ou a peculiaridades do próprio entrevistado, devem ser explicitados os procedimentos adotados para se corrigir ou amenizar estes erros.

Para suprir essa deficiência metodológica, o objetivo principal deste artigo é descrever um método para a correção das horas de sono inconsistentes. Os objetivos secundários são: analisar o padrão das respostas sobre horas de sono segundo algumas características sociodemográficas e relacionadas à rotina escolar (turno e tipo de escola) e analisar a concordância das respostas dos adolescentes que participaram do Estudo de Riscos Cardiovasculares em Adolescentes (ERICA) com a dos pais/responsáveis. 


\section{Métodos}

O ERICA é um estudo de delineamento transversal, multicêntrico, nacional, de base escolar, que visou a determinar a magnitude de diabetes e outros fatores de risco cardiovascular em adolescentes de cidades brasileiras com mais de 100 mil habitantes.

A população de pesquisa do ERICA correspondeu ao conjunto de adolescentes de 12 a 17 anos, que não possuíam qualquer deficiência temporária ou definitiva, não grávidas e que cursavam um dos três últimos anos do Ensino Fundamental ou dos três anos do Ensino Médio nos turnos da manhã ou da tarde, em escolas públicas ou privadas, urbanas ou rurais, localizadas em um dos 273 municípios com mais de 100 mil habitantes. A população de pesquisa apresenta representatividade nacional, das cinco macrorregiões brasileiras (Norte, Nordeste, Centro-oeste, Sudeste e Sul) e das capitais. Foram selecionadas escolas em 124 municípios, correspondendo a um total de 3.753 turmas, em 1.251 escolas. Em cada escola foram selecionadas três turmas. Nas turmas selecionadas, todos os alunos foram convidados a participar da pesquisa, mas somente os elegíveis foram analisados. Maiores informações sobre o delineamento amostral podem ser obtidas em Vasconcellos et al. 24.

A coleta dos dados ocorreu em 2013 e 2014. Consistiu na aplicação de um questionário autopreenchível, em um coletor eletrônico de dados denominado personal digital assistants (PDAs), para a coleta de medidas antropométricas, de pressão arterial, de consumo alimentar via recordatório de 24 horas e coleta de sangue para a avaliação bioquímica. Detalhes sobre a logística de coleta de dados podem ser obtidos em Bloch et al. 25.

O questionário autopreenchível continha informações sobre sexo, idade, cor da pele, além de dados sobre tabagismo, consumo de álcool, comportamento alimentar, atividade laboral, atividade física, de comportamento sedentário, saúde bucal, morbidade referida, sintomas depressivos e horas de sono.

O bloco do sono era composto por quatro perguntas. $\mathrm{O}$ adolescente deveria selecionar a hora em que ele costuma dormir e acordar em um dia de semana comum e no final de semana. As respostas eram fechadas, com 24 opções, uma para cada hora do dia, como mostradas em: http://www.erica. ufrj.br/media/arquivos/questionario\%20aluno\%20pda.pdf (páginas 31 e 32).

Foi entregue aos pais/responsáveis pelos alunos um questionário impresso autopreenchível para a coleta de informações sobre peso ao nascer do adolescente, questões relacionadas à amamentação, morbidade referida na família, dentre outras questões, além de perguntas sobre os horários habituais de dormir a acordar dos adolescentes, utilizando neste bloco específico do sono o mesmo questionário preenchido pelos adolescentes, como mostrado em: http://www.erica.ufrj.br/media/arquivos/ Questionario\%20responsavel\%20final.pdf (página 5).

A análise das inconsistências levou em consideração a distribuição das frequências das respostas dos alunos em relação aos horários de dormir e acordar, durante a semana e no final de semana, segundo o turno escolar. Os horários mais frequentes $(\geq 10 \%)$ foram considerados "Prováveis". Frequências inferiores a $10 \%$ foram analisadas baseando-se na incompatibilidade dos horários de dormir e acordar durante a semana no mesmo horário do turno de estudo (manhã de $8 \mathrm{~h}$ às $12 \mathrm{~h}$ e tarde de $13 \mathrm{~h}$ às $17 \mathrm{~h}$ ) e na pouca probabilidade do sono em horários matutinos ou vespertinos em substituição ao sono noturno. Nesses casos, alterações das horas de acordar e dormir foram feitas apenas quando considerou-se possível ter ocorrido troca da hora de dormir pela hora de acordar, ou vice-versa, tanto durante a semana quanto no final de semana, de acordo com a maior probabilidade das frequências de horários observada na amostra. Por exemplo, um aluno do turno da manhã que registrou a hora de acordar sendo $6 \mathrm{~h}$ da manhã e a hora de dormir $9 \mathrm{~h}$ da manhã, interpretou-se como sendo a hora de dormir $9 \mathrm{~h}$ da noite e a hora de acordar 6h da manhã.

A segunda etapa, após as correções aplicadas nos horários de dormir e acordar quando possível e o descarte dos horários improváveis, consistiu na obtenção da duração do sono com base na diferença, em horas, entre o início e o fim do sono, relatado como o intervalo compreendido entre ir para cama para dormir à noite e o despertar na manhã seguinte, tanto para durante a semana quanto para o final de semana. A duração de sono foi desconsiderada na análise de dados quando durante a semana e/ou final de semana o adolescente dormia $\leq 4$ ou $\geq 14$ horas.

A duração da média semanal do sono foi calculada como a média ponderada da hora de sono durante a semana e do final de semana, pela fórmula: (duração do sono durante a semana x $5+$ dura- 
ção do sono no final de semana x 2)/7 6,26,27, considerando as correções realizadas nos horários de dormir e acordar.

Foi criada uma variável para indicar que foram realizadas alterações nos horários de dormir e acordar, durante a semana e no final de semana. Essa variável indica três situações: (i) "Horários e duração válidos": os horários de dormir e acordar durante a semana e de dormir e acordar no final de semana não sofreram alterações, sendo considerados prováveis, e a duração do sono (durante a semana ou no final de semana) também foi considerada provável ( $\geq 4 \mathrm{~h}$ e $\leq 14 \mathrm{~h}$ ); (ii) "Horários alterados com duração do sono entre 4h e 14h": os horários de dormir e acordar durante a semana e de dormir e acordar no final de semana sofreram alterações, e a duração do sono (durante a semana ou no final de semana) foi considerada provável ( $\geq 4 \mathrm{~h}$ e $\leq 14 \mathrm{~h}$ ); (iii) "Horário de dormir ou de acordar ou duração improváveis": horário de dormir/acordar durante a semana e/ou de dormir/acordar no final de semana improváveis e sem critérios para correção e/ou duração do sono improvável foram excluídos da análise.

Inicialmente, foram estimados os percentuais e respectivos intervalos de 95\% de confiança (IC95\%) entre aqueles com respostas válidas (originais), corrigidas e excluídas da análise de acordo com sexo e idade dos adolescentes, tipo de escola (pública ou privada) e região do país. Posteriormente, foram comparadas as médias de duração do sono (válidas, corrigidas e excluídas) e respectivos IC95\%, também de acordo com essas mesmas características.

Foram comparadas as respostas originais e corrigidas dos alunos com a dos pais/responsáveis utilizando-se o coeficiente de correlação intraclasse e a distribuição da frequência da diferença entre as respostas originais e corrigidas dos alunos.

As análises foram realizadas com o pacote estatístico Stata 14.0 (https://www.stata.com). As médias ajustadas segundo o delineamento amostral 28 foram obtidas com base em rotinas estatísticas para amostragem complexa, Survey (svy).

O ERICA foi aprovado pelo Comitê de Ética em Pesquisa do Instituto de Estudos em Saúde Coletiva, Universidade Federal do Rio de Janeiro (IESC/UFRJ), em 2008 (processo no 45/2008). Também foi aprovada por esse Comitê a não obrigatoriedade de Termo de Consentimento Livre e Esclarecido (TCLE) para os adolescentes que aceitassem responder ao questionário no PDA e realizar coleta de medidas não invasivas, bastando assinar apenas um Termo de Assentimento. A obrigatoriedade do TCLE (com a assinatura dos pais/responsáveis e dos próprios alunos) foi mantida para aqueles que realizaram coleta de sangue.

\section{Resultados}

Das 1.251 escolas selecionadas na amostra original, 139 foram substituídas por escolas semelhantes (em tamanho, anos e turnos, e área geográfica) por se recusarem a participar do estudo. Quatro escolas, três em São Paulo e uma no Amapá, não foram substituídas por não existirem estabelecimentos semelhantes que aceitassem participar. O número de alunos matriculados nas 1.247 escolas avaliadas pelo ERICA foi de 114.162. Desses, 102.327 foram considerados elegíveis (faixa etária de 12 a 17 anos, não grávidas e não deficientes), sendo que $72,9 \%$ responderam ao questionário 29 , correspondendo a 74.589 adolescentes.

A Tabela 1 apresenta a distribuição das respostas originais dos alunos sobre os horários de acordar e dormir durante a semana e no final de semana, para os alunos dos turnos da manhã e da tarde. Os alunos que estudam no turno da tarde dormem e acordam mais tarde do que os que estudam no turno da manhã durante a semana. Ainda durante a semana, quase $50 \%$ de todos os alunos relataram dormir entre $23 \mathrm{~h}$ e $24 \mathrm{~h}$ e $8,9 \%$ dos alunos do turno da manhã entre $1 \mathrm{~h}$ e $2 \mathrm{~h}$ da manhã sendo este percentual de $19,6 \%$ para os alunos do turno da tarde. Já no final de semana, todos os alunos dormem e acordam mais tarde.

Durante a semana, para os 53.353 alunos do turno da manhã (71,5\%), a correção dos horários de dormir e acordar levou em consideração o horário escolar de $8 \mathrm{~h}$ às $12 \mathrm{~h}$. Os horários de dormir entre $20 \mathrm{~h}$ e $2 \mathrm{~h}$ foram assinalados por $95,4 \%$ dos alunos e este foi o período classificado como viável. Os horários de dormir entre $8 \mathrm{~h}$ e $14 \mathrm{~h}(1,5 \%)$ foram convertidos para as respectivas horas no turno da 


\section{Tabela 1}

Distribuição dos horários de acordar e dormir durante a semana e no final de semana, da amostra original, por turno. Estudo de Riscos Cardiovasculares em Adolescentes (ERICA), 2013-2014.

\begin{tabular}{|c|c|c|c|c|c|c|c|c|c|c|c|c|}
\hline \multirow[t]{4}{*}{ Horário } & \multicolumn{8}{|c|}{ Durante a semana } & \multirow{2}{*}{\multicolumn{4}{|c|}{$\begin{array}{l}\text { Final de semana } \\
\text { Manhã e tarde }\end{array}$}} \\
\hline & \multicolumn{4}{|c|}{ Manhã } & \multicolumn{4}{|c|}{ Tarde } & & & & \\
\hline & \multicolumn{2}{|c|}{ Dormir } & \multicolumn{2}{|c|}{ Acordar } & \multicolumn{2}{|c|}{ Dormir } & \multicolumn{2}{|c|}{ Acordar } & \multicolumn{2}{|c|}{ Dormir } & \multicolumn{2}{|c|}{ Acordar } \\
\hline & $\mathbf{n}$ & $\%$ & $\mathbf{n}$ & $\%$ & $\mathbf{n}$ & $\%$ & $\mathbf{n}$ & $\%$ & $\mathbf{n}$ & $\%$ & $\mathbf{n}$ & $\%$ \\
\hline $1 \mathrm{~h}$ & 3.395 & 6,4 & 38 * & 0,1 * & 2.560 & 12,1 & 20 * & 0,1 * & 11.749 & 15,8 & $147 * *$ & $0,2 * *$ \\
\hline $2 \mathrm{~h}$ & 1.359 & 2,5 & 25 * & 0,0 * & 1.589 & 7,5 & 16 * & 0,1 * & 9.865 & 13,2 & $90 * *$ & 0,1 ** \\
\hline $3 \mathrm{~h}$ & 495 * & 0,9 * & 29 * & 0,1 * & 849 & 4,0 & 26 * & 0,1 * & 6.864 & 9,2 & $103 * *$ & $0,1 * *$ \\
\hline $4 \mathrm{~h}$ & 102 * & 0,2 * & 418 * & 0,8 * & 232 & 1,1 & 118 * & 0,6 * & 2.899 & 3,9 & $215 *$ & 0,3 * \\
\hline $5 \mathrm{~h}$ & 78 * & 0,1 * & 8.062 & 15,1 & 88 * & 0,4 * & 632 & 3,0 & 1.517 & 2,0 & 479 & 0,6 \\
\hline $6 \mathrm{~h}$ & 106 * & 0,2 * & 33.615 & 63,0 & 37 * & 0,2 * & 2.100 & 9,9 & 547 & 0,7 & 2.103 & 2,8 \\
\hline $7 \mathrm{~h}$ & 33 * & 0,1 * & 5.108 & 9,6 & 19 * & 0,1 * & 2.486 & 11,7 & 204 * & 0,3 * & 4.411 & 5,9 \\
\hline $8 \mathrm{~h}$ & $31 * *$ & $0,1 * *$ & 654 * & 1,2 * & $18 * *$ & $0,1 * *$ & 3.741 & 17,6 & $119 * *$ & 0,2 ** & 9.291 & 12,5 \\
\hline $9 \mathrm{~h}$ & $70 * *$ & 0,1 ** & 772 * & 1,4 * & 48 ** & 0,2 ** & 4.306 & 20,3 & 137 ** & 0,2 ** & 12.839 & 17,2 \\
\hline $10 \mathrm{~h}$ & 177 ** & 0,3 ** & 857 * & 1,6 * & $73 * *$ & $0,3 * *$ & 3.594 & 16,9 & 227 ** & 0,3 ** & 14.978 & 20,1 \\
\hline $11 \mathrm{~h}$ & 150 ** & 0,3 ** & 680 * & 1,3 * & 70 ** & 0,3 ** & 2.086 & 9,8 & $307 * *$ & 0,4 ** & 10.840 & 14,5 \\
\hline $12 \mathrm{~h}$ & 87 ** & 0,2 ** & 386 * & 0,7 * & $52 * *$ & 0,2 ** & 714 & 3,4 & 267 ** & 0,4 ** & 8.555 & 11,5 \\
\hline $13 \mathrm{~h}$ & 160 ** & 0,3 ** & 141 * & 0,3 * & $34 * *$ & $0,2 * *$ & 77 * & 0,4 * & $179 * *$ & $0,2 * *$ & 3.224 & 4,3 \\
\hline $14 \mathrm{~h}$ & 120 ** & 0,2 ** & 83 * & 0,2 * & 25 ** & 0,1 ** & 34 * & 0,2 * & 170 ** & 0,2 ** & 1.785 & 2,4 \\
\hline $15 \mathrm{~h}$ & $56 *$ & 0,1 * & $43 *$ & 0,1 * & $18 * *$ & $0,1 * *$ & 19 * & 0,1 * & 108 ** & $0,1 * *$ & 872 & 1,2 \\
\hline $16 \mathrm{~h}$ & 18 * & 0,0 * & 73 * & 0,1 * & $3 * *$ & 0,0 ** & 14 * & 0,1 * & 44 ** & 0,1 ** & 341 * & 0,5 * \\
\hline $17 \mathrm{~h}$ & 31 * & 0,1 * & $290 * *$ & $0,5 * *$ & 13 * & 0,1 * & $26 * *$ & $0,1 * *$ & $55 * *$ & $0,1 * *$ & 149 ** & 0,2 ** \\
\hline $18 \mathrm{~h}$ & 323 * & 0,6 * & 1.332 ** & $2,5 * *$ & 97 * & 0,5 * & 118 ** & 0,6 ** & $209 * *$ & 0,3 ** & 215 ** & 0,3 ** \\
\hline $19 \mathrm{~h}$ & 410 * & 0,8 * & 273 ** & $0,5 * *$ & 108 * & 0,5 * & 172 ** & 0,8 ** & 224 * & 0,3 * & 307 ** & 0,4 ** \\
\hline $20 \mathrm{~h}$ & 1.645 & 3,1 & 63 * & 0,1 * & 476 & 2,2 & 217 ** & $1,0 * *$ & 627 & 0,8 & 699 ** & 0,9 ** \\
\hline $21 \mathrm{~h}$ & 5.162 & 9,7 & 88 * & 0,2 * & 1.206 & 5,7 & 298 ** & 1,4 ** & 1.963 & 2,6 & 1.007 ** & 1,4 ** \\
\hline $22 \mathrm{~h}$ & 13.043 & 24,4 & 119 * & 0,2 * & 3.431 & 16,2 & 205 ** & $1,0 * *$ & 5.706 & 7,7 & 1.025 ** & 1,4 ** \\
\hline $23 \mathrm{~h}$ & 15.688 & 29,4 & 109 * & 0,2 * & 5.134 & 24,2 & 143 ** & 0,7 ** & 12.369 & 16,6 & 633 ** & 0,8 ** \\
\hline $24 \mathrm{~h}$ & 10.614 & 19,9 & 95 * & 0,2 * & 5.056 & 23,8 & $74 * *$ & 0,3 ** & 18.233 & 24,4 & $281 * *$ & 0,4 ** \\
\hline Total & 53.353 & 100,0 & 53.353 & 100,0 & 21.236 & 100,0 & 21.236 & 100,0 & 74.589 & 100,0 & 74.589 & 100,0 \\
\hline
\end{tabular}

* Horário convertido para missing (ignorado);

** Reclassificação do horário diurno para noturno e vice-versa. Exemplo: horário de dormir dos alunos do turno da manhã que assinalaram 8h foi convertido para $20 \mathrm{~h}$.

noite (20h e $2 \mathrm{~h})$. Os horários entre $3 \mathrm{~h}$ e $7 \mathrm{~h}(1,5 \%)$ e entre $15 \mathrm{~h}$ e $19 \mathrm{~h}(1,6 \%)$ foram considerados improváveis e, portanto, excluídos da análise $(3,1 \%)$.

O horário viável de acordar entre os alunos do turno da manhã, em função de uma maior frequência no horário declarado, variou de $5 \mathrm{~h}$ às $7 \mathrm{~h}(87,7 \%)$. Horários de acordar entre $17 \mathrm{~h}$ e $19 \mathrm{~h}$ (3,5\%) foram convertidos para as respectivas horas no turno da manhã ( $5 \mathrm{~h}$ às $7 \mathrm{~h}$ ). Os horários entre $8 \mathrm{~h}$ e $16 \mathrm{~h}$ $(6,9 \%), 20 \mathrm{~h}$ e $24 \mathrm{~h}(0,9 \%)$ e $1 \mathrm{~h}$ e $4 \mathrm{~h}$ (1\%) foram considerados improváveis e excluídos da análise $(8,8 \%)$.

Durante a semana, para os 21.236 alunos do turno da tarde (28,5\%), a correção dos horários de dormir e acordar levou em consideração o horário escolar de $13 \mathrm{~h}$ às $17 \mathrm{~h}$. Para esses, foram considerados como viáveis os horários entre $20 \mathrm{~h}$ e $4 \mathrm{~h}(96,7 \%)$. Como possíveis horários de dormir com marcação trocada, os horários de $8 \mathrm{~h}$ às $16 \mathrm{~h}(1,5 \%)$ foram convertidos para $20 \mathrm{~h}$ às $4 \mathrm{~h}$. Foram considerados improváveis e excluídos da análise os horários para dormir de $5 \mathrm{~h}$ às $7 \mathrm{~h}(0,7 \%)$ e de $17 \mathrm{~h}$ às $19 \mathrm{~h}(1,1 \%)$, uma vez que a frequência nestes horários foi extremamente pequena, pouco provável de estar correta em função do horário escolar e improvável ter ocorrido marcação trocada.

Para os horários de acordar, foram considerados como viáveis os horários entre $5 \mathrm{~h}$ e $12 \mathrm{~h}(92,4 \%)$ e como possíveis horários com marcação trocada, de $17 \mathrm{~h}$ às $24 \mathrm{~h}$ (5,9\%), convertidos para horários de 


\section{Figura 1}

Fluxograma para avaliação de respostas consistentes e inconsistentes nas perguntas sobre horas de sono do questionário do aluno. Estudo de Riscos Cardiovasculares em Adolescentes (ERICA), 2013-2014.

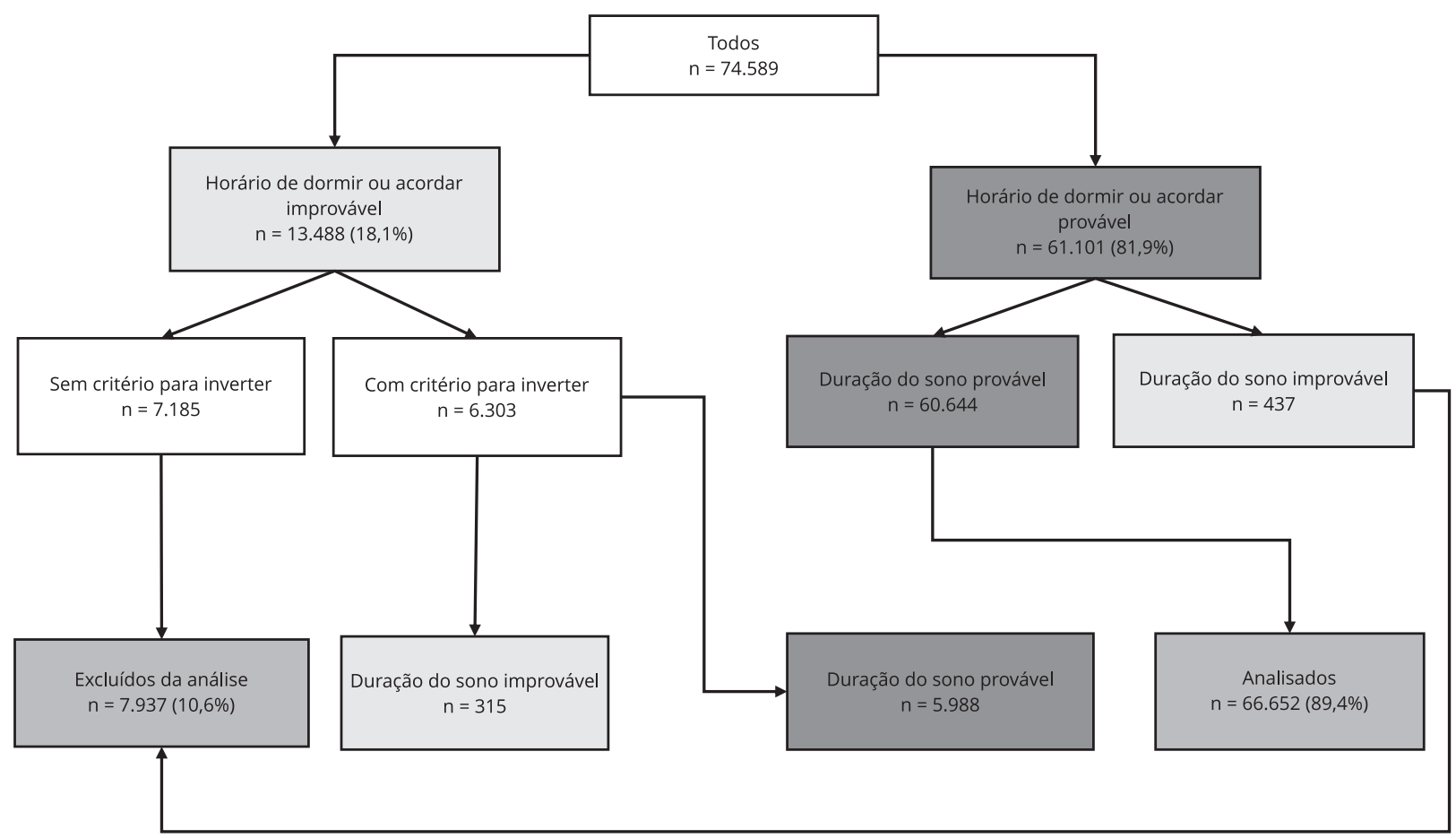


A Tabela 2 mostra a distribuição das diferentes respostas (válidas, corrigidas ou excluídas) pelas categorias das características sociodemográficas. Os homens tiveram mais respostas excluídas do que as mulheres e estas tiveram mais respostas válidas do que homens. Adolescentes com 16-17 anos apresentaram mais respostas válidas do que os de 14-15 anos e não houve diferenças destes grupos com o dos mais novos, 12-13 anos. Alunos de escolas privadas apresentaram mais respostas válidas do que os de escolas públicas, e os de escolas públicas registraram mais respostas corrigidas e excluídas do que os de escolas privadas. O maior percentual de respostas válidas foi o da Região Sul e o menor o da Região Norte. Essa região registrou mais respostas corrigidas do que as regiões Sudeste e Sul. As regiões Sudeste e Norte apresentaram os maiores porcentuais de respostas excluídas, e a Região Sul, o mais baixo.

A Tabela 3 compara as médias da duração de sono por sexo, faixa etária, turno de estudo, natureza administrativa da escola e região, ajustadas segundo o delineamento amostral, dos adolescentes cujas horas de dormir/acordar não foram alteradas, dos que foram alterados (pós-alteração) e da população total após a correção dos horários. Para todas as médias considerou-se que a duração do sono variou de 4 a 14 horas. Observa-se que as médias da duração de sono no grupo corrigido são um pouco maiores do que as dos demais grupos, porém não existem diferenças significativas para todas as categorias das características analisadas.

O questionário do responsável foi entregue por $60 \%$ dos adolescentes e foram comparadas as respostas dos responsáveis sobre a hora de dormir/acordar com as dos próprios adolescentes. Não foi observada uma boa correlação entre as respostas quando analisados os coeficientes de correlação intraclasse (Tabela 4), principalmente para a comparação entre as respostas originais dos adolescentes de todas as horas de sono questionadas (coeficiente variando de 0,26 a 0,55). A correção das respostas improváveis dos alunos produziu melhora nos coeficientes, variando de 0,46 a 0,61, para a hora de acordar no final de semana e durante a semana, respectivamente. $\mathrm{O}$ maior coeficiente de correlação foi o da hora de acordar durante a semana pós-correção.

Tabela 2

Comparação das características sociodemográficas dos adolescentes com respostas de horas e duração do sono válidas, corrigidas e as que se tornaram inválidas *. Estudo de Riscos Cardiovasculares em Adolescentes (ERICA), 2013-2014.

\begin{tabular}{|c|c|c|c|c|c|c|}
\hline \multirow[t]{2}{*}{ Características } & \multicolumn{2}{|c|}{ Dados originais } & \multicolumn{2}{|c|}{ Dados corrigidos } & \multicolumn{2}{|c|}{ Dados excluídos } \\
\hline & $\%$ & IC95\% & $\%$ & IC95\% & $\%$ & IC95\% \\
\hline \multicolumn{7}{|l|}{ Sexo } \\
\hline Feminino & 83,2 & $82,0-84,3$ & 7,7 & $7,1-8,3$ & 9,1 & $8,1-10,3$ \\
\hline Masculino & 77,1 & $75,7-78,4$ & 8,4 & $7,8-9,0$ & 14,6 & $13,3-15,9$ \\
\hline \multicolumn{7}{|l|}{ Idade (anos) } \\
\hline $12-13$ & 79,9 & $77,8-81,7$ & 8,6 & $7,7-9,6$ & 11,5 & $10,1-13,1$ \\
\hline $14-15$ & 78,8 & $77,4-80,1$ & 8,2 & $7,6-8,9$ & 13,0 & $11,7-14,5$ \\
\hline $16-17$ & 81,9 & $80,4-83,4$ & 7,2 & $6,6-7,8$ & 10,9 & $9,7-12,3$ \\
\hline \multicolumn{7}{|l|}{ Tipo de escola } \\
\hline Pública & 78,7 & $77,6-79,8$ & 8,4 & $7,9-8,9$ & 12,9 & $11,9-14,0$ \\
\hline Privada & 86,6 & $85,5-87,7$ & 6,5 & $5,9-7,2$ & 6,9 & $6,2-7,2$ \\
\hline \multicolumn{7}{|l|}{ Região } \\
\hline Norte & 77,9 & $76,4-79,4$ & 9,5 & $8,9-10,1$ & 12,6 & $11,2-14,2$ \\
\hline Nordeste & 81,9 & $80,4-83,3$ & 8,6 & $7,8-9,4$ & 9,5 & $8,5-10,7$ \\
\hline Centro-oeste & 80,0 & $77,8-81,9$ & 9,3 & $8,2-10,5$ & 10,8 & $9,2-12,5$ \\
\hline Sudeste & 78,9 & $77,0-80,8$ & 7,5 & $6,9-8,3$ & 13,5 & $12,0-15,3$ \\
\hline Sul & 83,6 & $81,6-85,3$ & 7,5 & $6,6-8,5$ & 9,0 & $7,0-11,5$ \\
\hline
\end{tabular}

IC95\%: intervalo de 95\% de confiança.

* Análises ajustadas para o delineamento amostral do estudo. 
Tabela 3

Médias e intervalos de 95\% de confiança (IC95\%) da duração do sono por características sociodemográficas em três diferentes etapas do processo de ajuste dos dados de sono. Estudo de Riscos Cardiovasculares em Adolescentes (ERICA), 2013-2014.

\begin{tabular}{|c|c|c|c|c|c|c|}
\hline & \multicolumn{2}{|c|}{$\begin{array}{l}\text { Horários de dormir e acordar sem } \\
\text { alteração e duração do sono * } \\
\text { válida (a) } \\
(n=60.664)\end{array}$} & \multicolumn{2}{|c|}{$\begin{array}{l}\text { Horários de dormir e/ou acordar } \\
\text { corrigidos + duração do sono * } \\
\text { válida (b) } \\
(n=5.988)\end{array}$} & \multicolumn{2}{|c|}{$\begin{array}{l}\text { Duração de sono * } \\
\text { total pós-correção }(a+b) \\
(n=66.652)\end{array}$} \\
\hline & Média ** & IC95\% & Média ** & IC95\% & Média ** & IC95\% \\
\hline Total & 8,2 & $8,2-8,3$ & 8,4 & $8,3-8,5$ & 8,3 & $8,2-8,3$ \\
\hline \multicolumn{7}{|l|}{ Sexo } \\
\hline Feminino & 8,3 & $8,2-8,3$ & 8,3 & $8,2-8,5$ & 8,3 & $8,2-8,3$ \\
\hline Masculino & 8,2 & $8,2-8,3$ & 8,5 & $8,3-8,7$ & 8,3 & $8,2-8,3$ \\
\hline \multicolumn{7}{|l|}{ Idade (anos) } \\
\hline $12-13$ & 8,8 & $8,7-8,9$ & 8,9 & $8,7-9,1$ & 8,8 & $8,7-8,9$ \\
\hline $14-15$ & 8,2 & $8,1-8,3$ & 8,4 & $8,2-8,5$ & 8,2 & $8,1-8,3$ \\
\hline $16-17$ & 7,7 & $7,6-7,7$ & 7,9 & $7,7-8,0$ & 7,7 & $7,6-77$ \\
\hline \multicolumn{7}{|l|}{ Turno } \\
\hline Manhã & 7,8 & $7,7-7,8$ & 7,8 & $7,7-7,9$ & 7,8 & $7,7-7,8$ \\
\hline Tarde & 9,3 & $9,2-9,4$ & 9,4 & $9,2-9,6$ & 9,3 & $9,2-9,4$ \\
\hline \multicolumn{7}{|l|}{ Tipo de escola } \\
\hline Pública & 8,3 & $8,3-8,4$ & 8,5 & $8,4-8,6$ & 8,3 & $8,3-8,4$ \\
\hline Privada & 7,9 & $7,8-8,0$ & 7,9 & $7,8-8,0$ & 7,9 & $7,8-8,0$ \\
\hline \multicolumn{7}{|l|}{ Região } \\
\hline Norte & 8,4 & $8,3-8,5$ & 8,7 & $8,5-8,9$ & 8,5 & $8,3-8,6$ \\
\hline Nordeste & 8,4 & $8,3-8,5$ & 8,7 & $8,5-8,9$ & 8,4 & $8,3-8,5$ \\
\hline Centro-oeste & 8,2 & $8,1-8,3$ & 8,4 & $8,2-8,6$ & 8,2 & $8,2-8,4$ \\
\hline Sudeste & 8,1 & $8,0-8,2$ & 8,2 & $8,0-8,4$ & 8,2 & $8,1-8,3$ \\
\hline Sul & 8,3 & $8,2-8,4$ & 8,6 & $8,4-8,8$ & 8,3 & $8,2-8,5$ \\
\hline
\end{tabular}

* Considerada duração do sono entre 4 e 14 horas;

** Análises foram ajustadas para o delineamento amostral do estudo.

\section{Tabela 4}

Correlação das respostas originais dos alunos e das corrigidas com as respostas dos pais/responsáveis, para horários de acordar e dormir, durante a semana e no final de semana. Estudo de Riscos Cardiovasculares em Adolescentes (ERICA), 2013-2014.

\begin{tabular}{|c|c|c|c|c|}
\hline \multirow[t]{2}{*}{ Horário de... } & \multicolumn{2}{|c|}{$\begin{array}{l}\text { Respostas originais dos alunos com as dos pais/ } \\
\text { responsáveis }\end{array}$} & \multicolumn{2}{|c|}{$\begin{array}{c}\text { Respostas dos alunos corrigidas com as dos } \\
\text { pais/responsáveis }\end{array}$} \\
\hline & $\mathrm{CCl}$ & IC95\% & $\mathrm{CCl}$ & IC95\% \\
\hline Dormir durante a semana & 0,51 & $0,50-0,53$ & 0,51 & $0,50-0,52$ \\
\hline Acordar durante a semana & 0,29 & $0,28-0,31$ & 0,61 & $0,60-0,63$ \\
\hline Dormir no final de semana & 0,55 & $0,51-0,59$ & 0,55 & $0,51-0,59$ \\
\hline Acordar no final de semana & 0,26 & $0,24-0,27$ & 0,46 & $0,45-0,46$ \\
\hline
\end{tabular}

CCI: coeficiente de correlação intraclasse; IC95\%: intervalo de 95\% de confiança. 
A Tabela 5 representa, em percentual, as diferenças entre as respostas dos alunos e dos pais/ responsáveis. Observa-se que os maiores percentuais, para todas as horas de sono avaliadas, estão concentrados na concordância total entre as respostas dos alunos (corrigidas ou não) e dos pais/ responsáveis originais. Em termos percentuais, não houve muita diferença entre os horários dos alunos, originais e corrigidos, com os dos pais/responsáveis. Destaque apenas para a redução de 9,7\% (respostas originais) para 1,9\% (respostas dos alunos corrigidas) para o horário de acordar durante a semana. A correção diminuiu o percentual de subestimação das respostas dos pais/responsáveis em 2 horas ou mais em relação às respostas dos alunos. As respostas dos pais/responsáveis subestimam mais em termos percentuais o horário de dormir do que o horário de acordar.

\section{Discussão}

A análise da distribuição das frequências das respostas e as correções aplicadas em função da incompatibilidade do turno escolar e das possíveis inversões dos horários noturnos pelos matutinos de sono foram relevantes para a recuperação de $8,1 \%$ dos dados de horas de sono autorrelatados por adolescentes, reduzindo as perdas de $18,7 \%$ para $10,6 \%$.

A elaboração das perguntas de um instrumento para avaliação da duração de sono merece uma atenção especial na fase de planejamento do estudo. Com o objetivo de minimizar a possibilidade de ocorrência de problemas de compreensão que levassem a erros de respostas, teve-se cuidado no ERICA com a escolha dos itens e com a adequação das palavras, de modo a minimizar as dúvidas quanto ao entendimento das perguntas 30 .

Questionários longos aumentam a probabilidade de inacurácia das respostas, que podem acabar sendo subutilizadas ou excluídas da análise por serem inconsistentes 18. É possível que pelo grande

\section{Tabela 5}

Diferença das horas de dormir e acordar, durante a semana e no final de semana, considerando as respostas dos pais/responsáveis e dos alunos (originais e corrigidas). Estudo de Riscos Cardiovasculares em Adolescentes (ERICA), 2013-2014.

\begin{tabular}{|c|c|c|c|c|c|c|c|c|}
\hline & \multicolumn{2}{|c|}{ Dormir } & \multicolumn{2}{|c|}{ Acordar } & \multicolumn{2}{|c|}{ Dormir no final de semana } & \multicolumn{2}{|c|}{$\begin{array}{c}\text { Acordar no final de } \\
\text { semana }\end{array}$} \\
\hline & $\mathbf{n}$ & $\%$ & $\mathbf{n}$ & $\%$ & $\mathbf{n}$ & $\%$ & $\mathbf{n}$ & $\%$ \\
\hline \multicolumn{9}{|l|}{$\begin{array}{l}\text { Diferença entre as respostas dos pais/ } \\
\text { responsáveis e as respostas originais dos } \\
\text { alunos }\end{array}$} \\
\hline $\begin{array}{l}\text { Subestimação do responsável em } 2 \text { horas } \\
\text { ou mais }\end{array}$ & 2.943 & 6,8 & 4197 & 9,7 & 4.630 & 10,7 & 6.685 & 15,4 \\
\hline Subestimação do responsável em 1 hora & 7.092 & 16,3 & 2.800 & 6,5 & 7.283 & 16,8 & 6.978 & 16,1 \\
\hline Concordância total com o responsável & 24.664 & 56,8 & 30.823 & 71,2 & 19.780 & 45,5 & 20.061 & 46,2 \\
\hline Superestimação do responsável em 1 hora & 4.339 & 10,0 & 3.434 & 7,9 & 3.400 & 7,8 & 5.676 & 13,1 \\
\hline $\begin{array}{l}\text { Superestimação do responsável em } 2 \\
\text { horas ou mais }\end{array}$ & 4.415 & 10,1 & 2.048 & 4,7 & 8.359 & 19,2 & 4.012 & 9,2 \\
\hline \multicolumn{9}{|l|}{$\begin{array}{l}\text { Diferença entre as respostas dos pais/ } \\
\text { responsáveis e as respostas corrigidas dos } \\
\text { alunos }\end{array}$} \\
\hline $\begin{array}{l}\text { Subestimação do responsável em } 2 \text { horas } \\
\text { ou mais }\end{array}$ & 2.873 & 6,7 & 774 & 1,9 & 4.510 & 10,4 & 4.470 & 10,4 \\
\hline Subestimação do responsável em 1 hora & 7.121 & 16,8 & 2.808 & 6,9 & 7.361 & 17,0 & 7.314 & 16,9 \\
\hline Concordância total com o responsável & 24.732 & 58,2 & 31.808 & 77,9 & 19.997 & 46,2 & 21.198 & 49,1 \\
\hline Superestimação do responsável em 1 hora & 4.336 & 10,2 & 3.434 & 8,4 & 3.468 & 8,0 & 6.033 & 14,0 \\
\hline $\begin{array}{l}\text { Superestimação do responsável em } 2 \\
\text { horas ou mais }\end{array}$ & 3.438 & 8,1 & 1.990 & 4,9 & 7.939 & 18,4 & 4.168 & 9,6 \\
\hline
\end{tabular}


número de questões a serem respondidas e o bloco do sono no questionário do aluno do ERICA ser o penúltimo de um total de 11 , isto pode ter contribuído para o cansaço, levando à ocorrência de respostas inconsistentes dos adolescentes, embora o tempo médio de preenchimento do questionário tenha sido de aproximadamente 30 minutos.

O bloco de perguntas sobre o sono contém questões fechadas, com todas as opções de respostas oferecidas de forma explícita para os entrevistados, facilitando o preenchimento 18 e a análise de dados. Nas respostas, com base na experiência do piloto, optou-se por listar primeiro, nas perguntas sobre as horas de dormir, aquelas do período noturno (a partir das $18 \mathrm{~h}$ ) e nas perguntas sobre horas de acordar, aquelas do período matutino (a partir das $4 \mathrm{~h}$ ), baseado no sistema de 12 horas segundo turno manhã e tarde, permitindo uma visualização rápida das opções mais prováveis, uma vez que o sistema de 24 horas não se mostrou eficaz no estudo piloto. Mesmo assim, a impaciência natural dos adolescentes pode ter contribuído para reduzir a motivação/concentração para preencher corretamente o questionário 22,23.

Não há uma maneira padrão para se perguntar sobre a duração do sono em pesquisas, e pouco se sabe sobre a comparabilidade das diferentes formas já utilizadas 31 . Um aspecto positivo para uma maior acurácia na obtenção da duração do sono pode ter sido o fato de se perguntar a hora de dormir e de acordar, separadamente, para dias durante a semana e do final de semana e, assim, realizar o cálculo da duração do sono, e não perguntar ao adolescente quantas horas ele habitualmente dorme por noite. Outros estudos optaram pela mesma estratégia 32,33,34. Acredita-se que a indagação direta do tempo de duração do sono esteja mais suscetível ao viés de memória. Um estudo francês 33 adotou de forma similar as mesmas perguntas usadas no ERICA, mas reduziu as opções de respostas de 24 para 13 na hora habitual de dormir e para 15 na hora de acordar, durante a semana, mas com intervalos de meia em meia hora. No caso do ERICA, as 24 opções de respostas estavam visíveis, mas uma tela de aviso aparecia ao aluno sempre que uma resposta improvável era selecionada, permitindo ao adolescente confirmar ou não a sua resposta.

O sucesso de uma coleta de dados, com consequente acurácia nas informações obtidas, depende também do treinamento dos supervisores e examinadores. É função importante do supervisor explicar aos alunos a relevância do correto preenchimento de cada resposta para a pesquisa 23 . O treinamento dos supervisores foi realizado pela própria Coordenação Central do estudo. Todos os supervisores e examinadores preencheram o questionário, pelo menos uma vez, para conhecerem todas as perguntas e poder auxiliar os respondentes no momento da coleta de dados.

Mesmo com todos os cuidados adotados na fase de planejamento e treinamento, a análise da distribuição das respostas dos alunos indicou que existiam respostas inconsistentes $(18,7 \%)$ no bloco do sono em relação ao seu turno escolar, como por exemplo, um aluno que acordava às $10 \mathrm{~h}$ da manhã e estudava no período matutino. Essa observação propiciou uma análise mais profunda de todas as respostas, e não apenas o cálculo automático da duração do sono. Mudanças foram efetuadas quando se observava troca de hora de dormir pelo de acordar e vice-versa (por exemplo, acordar às $6 \mathrm{~h}$ da noite e dormir às $11 \mathrm{~h}$ da manhã). Essa troca nos horários levou em consideração a falta de atenção do aluno no momento de responder ao questionário, não se atentando para qual horário estava respondendo (dormir ou acordar).

Observou-se que tanto os alunos cujas respostas necessitaram de correção, como aqueles que foram excluídos por terem respostas improváveis são: mais jovens, predominantemente de escolas públicas e da Região Norte. Esses fatores podem estar relacionados a uma maior dificuldade em entender corretamente as perguntas do questionário e responder às questões de forma satisfatória no PDA.

Considerou-se como duração de sono real de 4 a 14 horas, sendo eliminadas as durações de sono que não estivessem contempladas neste intervalo, por considerarmos que seria fisiologicamente improvável um adolescente dormir menos de 4 horas ou mais de 14, todas as noites, já que as perguntas se referiam ao sono habitual.

Apesar da recomendação da Fundação Nacional do Sono dos Estados Unidos para estimar pontos de corte para a duração do sono em todo o ciclo de vida 35, é importante destacar que, além da idade, existem fatores ambientais e genéticos que são fundamentais para o estabelecimento do cronótipo (variabilidade individual dos relógios biológicos) 36 . Portanto, não há na literatura um valor ou intervalo de duração de sono que seja ideal para adolescentes, o que dificulta a análise e a comparabilidade dos 
resultados com outros estudos. O estabelecimento de pontos de corte deve, de preferência, ser motivado e embasado pela teoria, com a finalidade de identificar ou predizer subgrupos populacionais 23 .

Para avaliar se as respostas dos alunos eram consistentes com as respostas de seus pais/responsáveis, foi realizada a comparação entre as respostas via coeficiente de correlação intraclasse, que indicou uma melhora no coeficiente quando se comparava com as respostas corrigidas dos alunos, principalmente para a hora de acordar. A hora de acordar foi melhor correlacionada entre pais/ responsáveis e alunos porque muitos pais ainda acordam seus filhos para ir à escola, diferentemente de se conhecer o horário de dormir. Os adolescentes estão expostos às influências eletrônicas como telefones celulares, computadores e videogames, o que faz com que os pais dificilmente consigam monitorar as horas dedicadas ao sono de seus filhos. A tendência de ir para cama tarde, como mostrado nos adolescentes do ERICA em que $65 \%$ dormem depois das $23 \mathrm{~h}$ (dado não apresentado), também ocorre em outras culturas 37,38 .

Ressalta-se que o valor mais elevado do coeficiente de correlação é menor do que 0,70 , sugerindo que a resposta dos pais pode não ser uma boa alternativa de informação a ser utilizada em estudos com adolescentes.

Portanto, se os $40 \%$ de pais que não retornaram os questionários têm percepções diferentes sobre a duração de sono de seus filhos, a concordância poderia ser ainda menor, justificando a decisão de não substituir as respostas dos alunos pelas dos pais, apesar das respostas dos alunos serem imprecisas.

Foram observadas algumas diferenças sociodemográficas entre alunos que entregaram e não entregaram o questionário do responsável, mas estas diferenças não foram estatisticamente significantes (dados não apresentados), levando a crer que é pouco provável que tenha ocorrido viés por perda de informações.

As médias da duração do sono após as correções e sem as correções foram muito similares. Embora o impacto das correções tenha sido pequeno na média da duração do sono, a correção evitou a exclusão da análise de 5.988 adolescentes e é provável que tenha reduzido o erro de classificação da duração do sono. Como a associação de diferentes características da população, inclusive dos fatores de risco cardiovascular com a duração do sono, será analisada em outros estudos, é importante que possíveis fontes de viés sejam reduzidas.

Apesar das limitações inerentes ao método usado no ERICA para a obtenção da duração do sono, a análise cuidadosa da distribuição das respostas permitiu que um porcentual importante fosse corrigido e utilizado. Mesmo assim, 10,6\% das respostas foram consideradas improváveis, mesmo após a correção, e foram excluídas, inclusive as horas de dormir/acordar isoladas e/ou a duração do sono.

Este estudo chama a atenção para a necessidade de explicitação de dois importantes aspectos - a qualidade do instrumento e o tratamento dado no momento da análise dos dados. A descrição detalhada das variáveis e dos indicadores utilizados, em especial dados oriundos de instrumentos autopreenchíveis, permite a avaliação de todo o processo pelo consumidor da informação, tornando-o crítico o suficiente para concordar ou não com as alterações propostas. A acurácia dos indicadores não só contribui para a validade interna de um estudo epidemiológico, mas também permite a comparação do estudo com achados obtidos em outras pesquisas 22,23.

Os dados apresentados indicam a necessidade de se analisar a priori as informações provenientes de autorrelatos de horas de acordar e dormir, para que estimativas corretas da duração do sono possam ser divulgadas. Este trabalho poderá estimular pesquisadores dedicados ao estudo do sono a analisar e corrigir possíveis inconsistências nas respostas obtidas com o uso de questionários/ entrevistas. Não identificamos na literatura nenhuma análise e descrição semelhantes empregadas na correção das horas de sono avaliadas em estudos observacionais, especialmente os que utilizem autorrelato e com adolescentes.

Dessa forma, o trabalho contribui para o aprimoramento da construção de instrumentos de coleta de dados em estudos observacionais, tornando transparente a forma de lidar com as limitações inerentes ao método de coleta de dados. 


\section{Colaboradores}

G. A. Abreu contribuiu com a concepção do artigo, análise, interpretação e preparação do manuscrito. T. L. N. Silva contribuiu com a análise e interpretação dos dados e revisão do manuscrito final. L. R. Teixeira contribuiu com a interpretação dos dados e revisão do manuscrito final. $\mathrm{K}$. V. Bloch contribuiu com a concepção do artigo, análise, interpretação e revisão do manuscrito final.

\section{Informações adicionais}

ORCID: Gabriela de Azevedo Abreu (0000-00034420-9116); Thiago Luiz Nogueira da Silva (00000003-1961-1883); Liliane Reis Teixeira (00000003-2460-0767); Katia Vergetti Bloch (00000002-6992-3159).

\section{Agradecimentos}

Financiamento: Decit/SCTIE/MS, CT-Saúde/ MCTI, FINEP (01090421) e CNPq (565037/2010-2).

\section{Referências}

1. Wolfson AR, Carskadon MA. Sleep schedules and daytime functioning in adolescents. Child Dev 1998; 69:875-87.

2. Hitze B, Bosy-Westphal A, Bielfeldt F, Settler U, Plachta-Danielzik S, Pfeuffer M, et al. Determinants and impact of sleep duration in children and adolescents: data of the Kiel Obesity Prevention Study. Eur J Clin Nutr 2009; 63:739-46.

3. Pereira EF, Teixeira CS, Louzada FM. Sonolência diurna excessiva em adolescentes: prevalência e fatores associados. Rev Paul Pediatr 2010; 28:98-103.

4. Roberts R, Roberts CR, Duong H. Sleepless in adolescence: prospective data on sleep deprivation, health and functioning. J Adolesc 2009; 32:1045-57.

5. Gangwisch JE, Malaspina D, Babiss LA, Opler MG, Posner K, Shen S, et al. Short sleep duration as a risk factor for hypercholesterolemia: analyses of the National Longitudinal Study of Adolescent Health. Sleep 2010; 33:956-61.

6. Kong AP, Wing YK, Choi KC, Li AM, Ko GT, Ma RC, et al. Associations of sleep duration with obesity and serum lipid profile in children and adolescents. Sleep Med 2011; 12:659-65.

7. Marshall NS, Glozier N, Grunstein RR. Is sleep duration related to obesity? A critical review of the epidemiological evidence. Sleep Med Rev 2008; 12:289-98.

8. Lauderdale DS, Knutson KL, Yan LL, Liu K, Rathouz PJ. Sleep duration: how well do selfreports reflect objective measures? The CARDIA Sleep Study. Epidemiology 2008; 19:83845.

9. George NM, Davis JE. Assessing sleep in adolescents through a better understanding of sleep physiology. Am J Nurs 2013; 113:26-31.

10. Morgenthaler TI, Lee-Chiong T, Alessi C, Friedman L, Aurora RN, Boehlecke B, et al. Practice parameters for the clinical evaluation and treatment of circadian rhythm sleep disorders. An American Academy of Sleep Medicine report. Sleep 2007; 30:1445-59.

11. Owens JA. Update in pediatric sleep medicine. Curr Opin Pulm Med 2011; 17:425-30.

12. Toth LA, Jhaveri K. Sleep mechanisms in health and disease. Comp Med 2003; 53:47386.

13. Kushida CA, Littner MR, Morgenthaler T, Alessi CA, Bailey D, Coleman Jr. J, et al. Practice parameters for the indications for polysomnography and related procedures: an update for 2005. Sleep 2005; 28:499-521.

14. Morgenthaler T, Alessi C, Friedman L, Owens J, Kapur V, Boehlecke B, et al. Practice parameters for the use of actigraphy in the assessment of sleep and sleep disorders: an update for 2007. Sleep 2007; 30:519-29.

15. Lockley SW, Skene DJ, Arendt J. Comparison between subjective and actigraphic measurement of sleep and sleep rhythms. J Sleep Res 1999; 8:175-83. 
16. Weatherwax KJ, Lin X, Marzec ML, Malow BA. Obstructive sleep apnea in epilepsy patients: the Sleep Apnea scale of the Sleep Disorders Questionnaire (SA-SDQ) is a useful screening instrument for obstructive sleep apnea in a disease-specific population. Sleep Med 2003; 4:517-21.

17. Johns MW. Appendix I: the subjective measurement of excessive daytime sleepiness. In: Pandi-Perumal SR, Verster JC, Monti JM, Lader M, Langer SZ, editors. Sleep disorders: diagnosis and therapeutics. London: Informa Healthcare; 2008. p. 643-57.

18. Bloch KV, Klein $\mathrm{CH}$. Estudos seccionais. In: Medronho RA, Bloch KV, Luiz RR, Werneck GL, organizadores. Epidemiologia. São Paulo: Atheneu; 2009. p. 193-220.

19. Rothman KJ, Greenland S. Modern epidemiology. 2nd Ed. Philadelphia: Lippincott-Raven; 1998.

20. Miettinen O. Theoretical epidemiology: principles of occurrence research in medicine. New York: John Wiley \& Sons; 1985.

21. Kleimbaum DG, Kupper LL, Morgenstern H. Epidemiologic research: principles and quantitative methods. New York: John Wiley \& Sons; 1982.

22. Reichenheim ME, Moraes CL. Desenvolvimento de instrumentos de aferição epidemiológicos. In: Kac G, Sichieri R, Gigante DP, organizadores. Epidemiologia nutricional. Rio de Janeiro: Editora Fiocruz/Atheneu; 2009. p. 370-404.

23. Reichenheim ME, Moraes CL. Buscando a qualidade das informações em pesquisas epidemiológicas. In: Minayo MCS, Deslandes SF, organizadores. Caminhos do pensamento: epistemologia e método. Rio de Janeiro: Editora Fiocruz; 2013. p. 227-54.

24. Vasconcellos MTL, Silva PLN, Szklo M, Kuschnir MCC, Klein CH, Abreu GA, et al. Sampling design for the Study of Cardiovascular Risks in Adolescents (ERICA). Cad Saúde Pública 2015; 31:921-30.

25. Bloch KV, Szklo M, Kuschnir MC, Abreu GA, Barufaldi LA, Klein CH, et al. The study of cardiovascular risk in adolescents - ERICA: rationale, design and sample characteristics of a national survey examining cardiovascular risk factor profile in Brazilian adolescents. BMC Public Health 2015; 15:94.
26. Taheri S, Lin L, Austin D, Young T, Mignot E. Short sleep duration is associated with reduced leptin, elevated ghrelin, and increased body mass index. PLoS Med 2004; 1:e62.

27. Knutson KL, Ryden AM, Mander BA, Van Cauter E. Role of sleep duration and quality in the risk and severity of type 2 diabetes mellitus. Arch Intern Med 2006; 166:1768-74.

28. Skinner C, Holt D, Smith T. Analysis of complex surveys. Chichester: John Wiley \& Sons; 1989.

29. Silva TLN, Klein CH, Souza AM, Barufaldi LA, Abreu GA, Kuschnir MCC, et al. Response rate in the Study of Cardiovascular Risks in Adolescents - ERICA. Rev Saúde Pública 2016; 50 Suppl 1:3s.

30. Ximenes RAA, Araújo TVB. Validade interna em estudos de corte transversal: reflexões a partir de uma investigação sobre esquistossomose mansônica e condições socioeconômicas. Cad Saúde Pública 1995; 11:118-27.

31. Lauderdale DS. Survey questions about sleep duration: does asking separately about weekdays and weekends matter? Behav Sleep Med 2014; 12:158-68.

32. Maslowsky J, Ozer EJ. Developmental trends in sleep duration in adolescence and young adulthood: evidence from a national United States sample. J Adolesc Health 2014; 54:691-7.

33. Leger D, Beck F, Richard JB, Godeau E. Total sleep time severely drops during adolescence. PLoS One 2012; 7:e45204.

34. Kim SJ, Lee YJ, Cho SJ, Cho IH, Lim W. Relationship between weekend catch-up sleep and poor performance on attention tasks in Korean adolescents. Arch Pediatr Adolesc Med 2011; 165:806-12.

35. Hirshkowitz M, Whiton K, Albert SM, Alessi C, Bruni O, DonCarlos L, et al. National Sleep Foundation's sleep time duration recommendations: methodology and results summary. Sleep Health 2015; 1:40-3.

36. Roenneberg T, Kuehnle T, Pramstaller PP, Ricken J, Havel M, Guth A, et al. A marker for the end of adolescence. Curr Biol 2004; 14:R1038-9.

37. Chung KF, Cheung MM. Sleep-wake patterns and sleep disturbance among Hong Kong Chinese adolescents. Sleep 2008; 31:185-94.

38. Yang CK, Kim JK, Patel SR, Lee JH. Age-related changes in sleep/wake patterns among Korean teenagers. Pediatrics 2005; 115(1 Suppl):250-6. 


\section{Abstract}

The study aimed to assess sleep behavior in adolescents 12 to 17 years of age participating in ERICA (Study of Cardiovascular Risk Factors in Adolescents), based on sociodemographic characteristics, school shift, and type of school, and to describe the correction of inconsistent sleep times in a nationally representative study in Brazil. Data were collected in 2013 and 2014. Four questions were asked on habitual time for sleeping and waking on weekdays and weekends, with 24 possible answers, one for each hour of the day. Analysis of inconsistencies considered the distribution of frequencies of answers as to sleeping and waking times, in addition to compatibility with the school shift. Sleep duration during the week and on weekends was obtained by the difference between sleeping and waking times, and differences of $\leq 4$ or $\geq 14$ hours were excluded. Mean total sleep duration in seven days was calculated by the formula (weekday sleep duration $x 5+$ weekend sleep $d u$ ration $x 2) / 7$. The following groups were created: original data (answers that did not require correction), corrected data (inconsistent, but amenable to correction), and excluded data (inconsistent, and for which there was no criterion for correction). Correction recovered inconsistent information for 5,988 adolescents, $8 \%$ of the 74,589 participants. A total of 7,937 (10.6\%) answers were excluded. Adolescents whose information was corrected or excluded were younger, predominantly males, from public schools, and from the North of Brazil. Correction minimized losses and lent greater consistency to the data treatment. The study contributes to the improvement of data collection tools in observational studies, lending transparency to the way of dealing with inherent limitations in the data collection method.

Data Accuracy; Health Surveys; Sleep

\section{Resumen}

El objetivo fue evaluar el comportamiento del sueño en adolescentes de 12 a 17 años, participantes en el ERICA (Estudio de Riesgos Cardiovasculares en Adolescentes), según características sociodemográficas, turnos y tipos de escuela, donde además se describe la corrección de las horas incompatibles en un estudio de nivel nacional en Brasil. La recogida de datos se realizó en 2013 y 2014. Se hicieron cuatro preguntas sobre las horas habituales de sueño y de despertarse durante la semana y el fin de semana, con 24 opciones de respuesta, una para cada hora del día. El análisis de las incompatibilidades consideró la distribución de frecuencias en las respuestas, respecto a las horas habituales de sueño y de despertarse, además de la compatibilidad con el turno. La duración del sueño durante la semana y fin de semana se obtuvo mediante la diferencia entre horas habituales de sueño y de despertarse y fue excluida si $\leq 4 \hat{o}$ $\geq 14$ horas. La media de sueño total de la semana se calculó mediante la fórmula: (duración del sueño durante la semana $x 5+$ duración del sueño durante el fin de semana $x 2$ )/ 7 . Se crearon tres grupos: datos originales (respuestas que no necesitaron correcciones), datos corregidos (inconsistentes, pero plausibles de corrección) y datos excluidos (inconsistentes en los que no había criterio para la corrección). La corrección recuperó información inconsistente de 5.988 adolescentes (8\%) de los 74.589 participantes. Se excluyeron 7.937 (10,6\%) respuestas. Los adolescentes cuya información fue corregida o excluida son más jóvenes, sexo masculino, procedentes de escuelas públicas y de la región Norte. La corrección minimizó pérdidas y otorgó una mayor consistencia al tratamiento de los datos. El estudio contribuye al perfeccionamiento de la creación de instrumentos de recogida de datos en estudios observacionales, haciendo transparente la forma de enfrentarse a las limitaciones inherentes del método de recogida de datos.

Exactitud de los Datos; Encuestas

Epidemiológicas; Sueño
Recebido em 09/Ago/2018

Versão final reapresentada em 17/Jul/2019

Aprovado em 02/Ago/2019 